\title{
ANÁLISE DA VIABILIDADE ECONÔMICA DE UM PLANTIO DE TECA (Tectona grandis) NA FAZENDA CONFRARIA DO CAMPO, EM SANTARÉM-NOVO, PARÁ
}

\author{
Livia Karine Lima Rabelo', Sarah Rabelo Traian Baumann¹, Mayra Piloni Maestri2, Marina Gabriela \\ Cardoso de Aquino ${ }^{3}$, Camila Amorim Santa Brigida ${ }^{1}$, Lenilson Ferreira Palheta ${ }^{4}$
}

\footnotetext{
${ }^{1}$ Discente de Engenharia Florestal da Universidade Federal do Oeste do Pará; ${ }^{2}$ Doutoranda em Ciências Florestais da Universidade Federal Rural da Amazônia; ${ }^{3}$ Mestranda em Engenharia Florestal da Universidade do Estado de Santa Catarina; ${ }^{4}$ Mestre em Ciências Florestais pela Universidade Federal Rural da Amazônia.
}

RESUMO: As boas propriedades da madeira de teca lhe conferem múltiplas aplicações, sendo muito valorizada no mercado internacional como madeira nobre e de alto valor agregado. O presente trabalho teve como objetivo determinar os custos de um povoamento florestal de teca, assim como as receitas geradas, por meio da demonstrar dos custos e receitas de cada etapa do empreendimento em estudo e o retorno financeiro da atividade. A área de estudo está localizada no Município de Santarém-Novo-PA, sendo os dados originários de povoamento homogêneo de Teca (Tectona grandis L. f.), em arranjo de $3 \mathrm{~m} \times 4 \mathrm{~m}$, em área de 300 hectares. Foram avaliados, o investimento na implantação, manutenção, colheita e transporte do plantio, assim como a produtividade do povoamento. Foram utilizados os indicadores econômicos: Valor Presente Líquido (VPL); Valor Anual Equivalente (VAE); Razão Benefício Custo (RBC) e Taxa Interna de Retorno (TIR). Os dados utilizados neste estudo foram tabulados em planilhas do Microsoft Excel. O custo total do empreendimento foi de $15.796,00$, onde os custos na fase de implantação iguais a $R \$ 2.486,00$, custos de colheita $R \$ 10.970,00$ e de manutenção $R \$ 2.340,00$. Quando se trata das receitas do plantio, está foi de $R \$ 68.750,00$. Os valores encontrados com os indicadores econômicos apontam que, o plantio em questão possui viabilidade econômica, obtendo-se: $\quad \mathrm{VPL}=\mathrm{R} \$ 9.969,28 ; \quad \mathrm{VAE}=\mathrm{R} \$ 1.310,70 ; \quad \mathrm{TIR}=20 \%$ e $R B C=10,59$. A partir da análise de dados desse projeto, pode-se concluir que, o empreendimento é viável economicamente. No entanto, apesar de possuir altos valores de lucratividade, com baixos custos de implantação, o plantio só apresentou retorno financeiro no último ano.

Palavras-chave: Economia florestal, Produtividade, Silvicultura 


\section{ANALYSIS OF THE ECONOMIC VIABILITY OF TECA (Tectona grandis) PLANTATION ON THE CONFRARIA DO CAMPO FARM, IN SANTARÉM-NOVO, PARÁ}

ABSTRACT: The good properties of teca wood give it multiple applications, being highly valued in the international market as noble wood and of high added value. The purpose of this paper is to show how attractive and profitable the use of teca is in forest stands. The study area is located in the Municipality of Santarém-Novo-PA, with data originating from a homogeneous population of teca (Tectona grandis L. f.), In a $3 m x$ $4 \mathrm{~m}$ arrangement, in an area of 300 hectares. Investment in the implantation, maintenance, harvesting and transportation of the plantation was evaluated, as well as the productivity of the stand. Economic indicators were used: Net Present Value (NPV); Equivalent Annual Value (EAV); Cost Benefit Ratio (CBR) and Internal Rate of Return (IRR). The data used in this study were tabulated in Microsoft Excel spreadsheets. The total cost of the project was $R \$ 15,796.00$, with costs in the implementation phase equal to $R \$ 2,486.00$, harvest costs $R \$ 10,970.00$ and maintenance costs $R \$ 2,340.00$. When it comes to planting revenues, this was $R \$ 68,750.00$. The values found with the economic indicators indicate that the plantation in question has economic viability, obtaining: $N P V=R \$ 9,969.28 ; E A V=R \$ 1,310.70 ;$ I $R R=20 \%$ and $C B R=10.59$. From the data analysis of this project, it can be concluded that the project is economically viable. However, despite having high profitability values, with low implementation costs, planting only showed a financial return in the last year.

Keywords: Forest economy, Productivity, Forestry

\section{INTRODUÇÃO}

A Tectona grandis L.f., comumente conhecida como teca, é uma espécie de origem asiática, com distribuição natural na Índia, Mianmar, Tailândia e Laos, que foi introduzida em diversas regiões da África e América do Sul e Central, principalmente na Colômbia, Equador, El Salvador, Panamá, Trinidad e Tobago e Venezuela, devido a gama de utilidades que sua madeira apresenta, com produção variando de acordo com a adaptação às condições edafoclimáticas (NOCETTI, 2011).

Em ambientes naturais, a teca apresenta grande porte, podendo alcançar mais de 60 metros de altura. Em sua maioria possui tronco retilíneo, com seção circular e conicidade reduzida. A casca é gretada e de cor cinza ou marrom, e, embora não seja espessa, fornece elevada resistência ao fogo. Suas folhas despertam a atenção pelo tamanho, que pode alcançar $60 \times 80 \mathrm{~cm}$ de comprimento e $25 \mathrm{~cm}$ de largura, e por serem caducas, isto é, caírem por ocasião da estação seca (PELISSARI et al., 2014). 
Segundo o Instituto de Pesquisas e Estudos Florestais (2003), a teca apresenta vistoso contraste entre as cores do alburno amarelado ou esbranquiçado, geralmente estreito, e a cor marrom brilhante do cerne, o que ocasiona a procura da madeira desta espécie para decorações luxuosas. Isso ocorre porque ambos contêm uma substancia denominada caucho que, diminui infiltração de água e lubrifica a superfície. Apresenta anéis de crescimento nítidos e diferenciados nos cortes transversais e densidade média de $0,65 \mathrm{~g} / \mathrm{cm}^{3}$, considerada leve, todavia, de boa resistência a peso, tração e flexão (COSTA et al., 2012).

As boas propriedades da madeira de teca lhe conferem múltiplas aplicações, sendo muito valorizada no mercado internacional como madeira nobre e de alto valor agregado. Flórez (2015) escreveu sobre ensaios de simulação laboratorial com teca no $13^{\circ}$ ano de idade para uso em pisos de madeira e constatou que ela pode ser empregada em áreas com tráfego leve a moderado, sem execução de grandes esforços, podendo ser usada em residências e outros ambientes pequenos. Em outra vertente, Paes et al. (2015) caracterizou tecnologicamente a madeira de teca aos 7 anos para a produção de móveis e obteve bons resultados de estética e acabamento para o material confeccionado, evidenciando potencial uso na indústria moveleira.

Além disso, a madeira da espécie apresenta outras finalidades, tais como a construção naval, teares, andaimes, objetos para decoração e utensílios domésticos. As árvores de teca também são úteis como cercas vivas, as folhas são utilizadas como embalagens de açougue, extração de corantes para tingimento de seda e fármacos de combate à malária e anemia, somado a pesquisas recentes para o mercado de fixação de carbono e com extratos de flores que apontam o potencial antidiabético, antioxidante, anti-inflamatório, analgésico e de cicatrização de feridas (BHATIA et al., 2011; RAMACHANDRAN et al., 2011; KLIPPEL, 2013).

A teca é uma das espécies florestais de melhor aprovação no mercado internacional de produtos madeireiros, sobretudo pelas suas características de trabalhabilidade e durabilidade (MORA; HERNANDÉZ 2007). Segundo Castro et al., (2007), a produção mundial da madeira de Tectona grandis é de, aproximadamente, 3 milhões de $\mathrm{m}^{3} / \mathrm{ano}$, sendo que majoritariamente é consumida pelo mercado interno dos países produtores. No Brasil, verifica-se que os povoamentos de Tectona 
grandis L. f. apresentam produtividade extremamente variável. Assim, autores como Bezerra et al. (2011) buscaram analisar a viabilidade econômica de plantios desta espécie.

Dessa forma, o objetivo geral do presente estudo é de determinar os custos de um povoamento florestal de teca, assim como as receitas geradas, por meio da demonstrar dos custos e receitas de cada etapa do empreendimento em estudo e o retorno financeiro da atividade.

\section{MATERIAL E MÉTODOS}

Área de estudo

A área de estudo está situada no Município de Santarém Novo pertence à Microrregião de Bragantina, localizado as margens do Rio Maracanã, ocupa uma área de 229,510 km², fazendo limite ao Norte com o município de São João de Pirabas; ao Leste com o município de Primavera; mais ao Sul com os municípios de Peixe - Boi, Nova Timboteua; e a Oeste com o município de Maracanã. Sua população se encontra em torno de 6.341 habitantes. Suas coordenadas geográficas são: Latitude $00^{\circ} 55^{\prime}$ 44" Sul e Longitude 47 23' 49" Oeste, estando 31 metros acima do nível do mar (PREFEITURA MUNICIPAL DE SANTARÉM NOVO, 2018). 


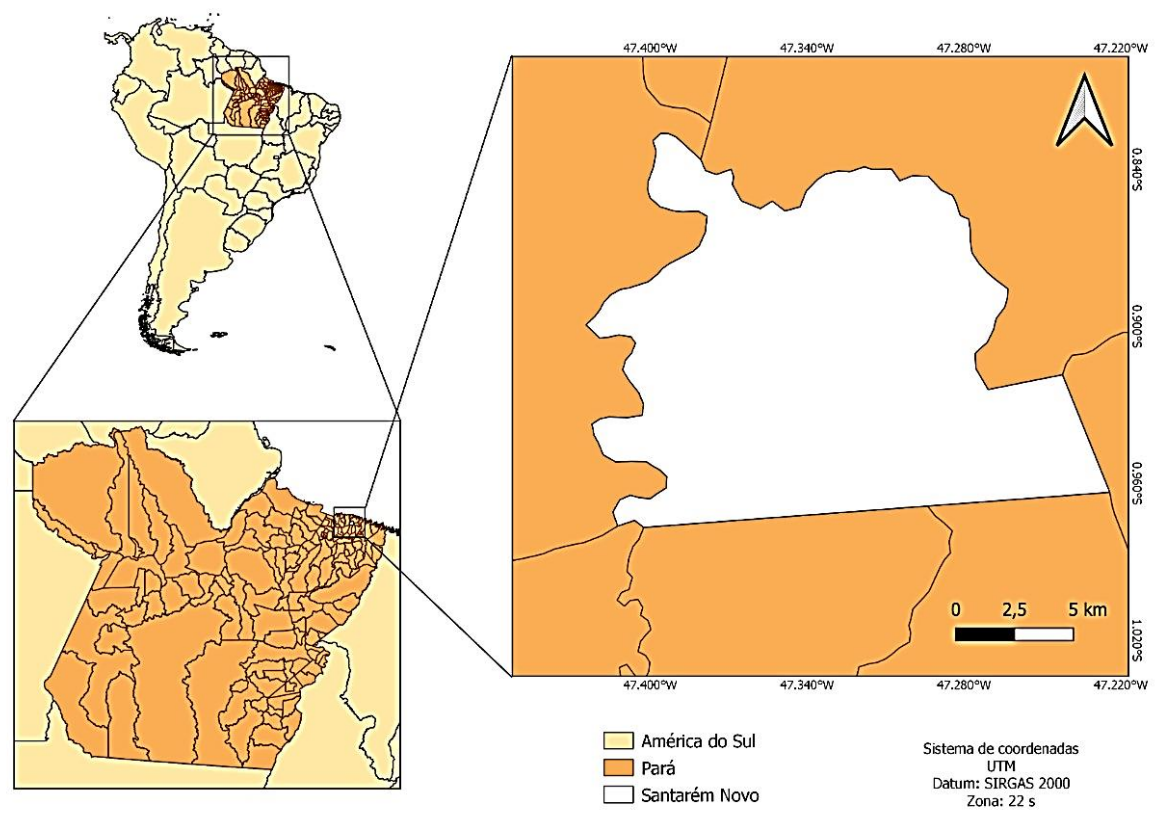

Figura 1. Localização do município de Santarém Novo-Pará. Fonte: Google Maps. Figure 1. Location of the municipality of Santarém Novo-Pará. Source: Google Maps.

Coleta e Tabulação de Dados

Os dados são pertencentes a povoamento homogêneo de Teca (Tectona grandis L. f.), plantados sob o arranjo de $3 \mathrm{~m} \times 4 \mathrm{~m}$, em uma área total de 300 hectares, acarretando em uma densidade populacional de 833 árv.ha-1.Os itens avaliados foram: investimento que o empreendedor teve em relação a implantação, manutenção e colheita do plantio; transporte, que é de responsabilidade da empresa que adquiri o produto; bem como, a produtividade do povoamento, com o corte realizado aos 16 anos. Foi realizada entrevista, ao proprietário da fazenda Confraria do Campo, onde o plantio está localizado. Os dados utilizados neste estudo foram tabulados em planilhas do Microsoft Excel, foram realizados cálculos, com objetivo de analisar os custos e receitas do plantio na área de um hectare e os indicadores econômicos que serão descritos mais abaixo. Para melhor visualização dos resultados, foram inseridas tabelas. 


\section{Custos e Receitas}

A mão-de-obra utilizada no manejo da área é a mais importante dentre os custos das atividades florestais. Dentre as atividades que requerem utilização da mão de obra, a limpeza da área, gradagem, plantio, adubação, colheita e transporte do produto final podem ser considerados as que geram maior custo.

No preparo da área foi contratado um operário para cada atividade, necessitando-se de seis diárias para capina manual. Já as atividades de combate às formigas, plantio e replantio, e gradagem foram realizadas pelo proprietário do empreendimento.

O corte raso foi realizado quando o plantio encontrava-se com 16 anos de idade. As toras após a colheita foram comercializadas com uma fábrica localizada no município de Dom Eliseu-PA. A variável transporte foi calculada a um custo fixo de $80,0 \mathrm{R} \$ / \mathrm{m}^{3}$ (oitenta reais por metro cubico), uma vez que, a própria indústria compradora das toras, era a responsável pelo transporte das mesmas da área do plantio até seu destino final.

Análise Econômica

Foi calculado o fluxo de caixa para visualização dos custos e receitas, bem como os lucros gerados ao longo do tempo de vida útil do empreendimento. De acordo com Martins e Oliveira (2018) o fluxo de caixa é o instrumento pelo qual as entradas e saídas são projetadas para períodos futuros, podendo ser utilizado para controle e tomada de decisão. Desse modo, foram calculados:

- Valor Presente Líquido (VPL): este é tido como um estimador consistente para cálculo de viabilidade econômica, pois o mesmo faz uma relação com o valor atual e o valor em que o empreendimento foi iniciado, descontando-se a taxa de juros determinada pelo mercado (SANGUINO, 2009). Quando seu resultado é positivo, o projeto em análise deve ser considerado viável economicamente, já quando negativo mostra-se inviável.

O VPL pode ser calculado de acordo com a fórmula abaixo:

$$
V P L=\sum_{i=0}^{n} \frac{B_{t}-C_{t}}{(1+i)^{t}}
$$


Em que: $V P L=$ Valor Presente Líquido; $B_{t}=$ Receita em cada período (ano) do projeto; $C_{t}=$ Custo em cada período (ano) do projeto; $\mathrm{n}$ = Número de anos do projeto ou período usado em cada atividade; $\mathrm{i}$ = Taxa de desconto (juros).

- Valor Anual Equivalente (VAE): neste indicador econômico o VPL é transformado em fluxo de receitas ou custos periódicos e contínuos, comparado ao valor atual, durante o tempo em que o projeto foi realizado. Se o VAE for maior que zero, este apresenta viabilidade econômica (VIRGENS et al., 2016).

O VAE pode ser calculado de acordo com a fórmula abaixo:

$$
V A E=\sum_{i=0}^{n} \frac{F C_{t}}{(1+i)^{t}} x\left[\frac{i x(1+i)^{t}}{(1+i)^{t}-1}\right]
$$

- Taxa Interna de Retorno (TIR): é a taxa de retorno anual do capital investido. O critério da TIR está associado a estudos de viabilidade econômica em que se busca verificar se a rentabilidade do empreendimento é superior, inferior ou igual ao custo do capital que será utilizado para financiar o projeto (REZENDE, 2006). Em relação ao critério de viabilidade, o projeto será viável quando a TIR for superior à Taxa Mínima de Atratividade (TMA). Algebricamente tem-se:

$$
T I R=\sum_{i=0}^{n} \cdot \frac{B_{t-C_{t}}}{\left(1+i^{*}\right)^{t}}
$$

Em que: $\mathrm{Bt}=$ Receita em cada período de tempo (ano) do projeto; $\mathrm{Ct}=$ Custo em cada período de tempo (ano) do projeto; $\mathrm{n}=$ Número de anos do projeto ou período de tempo usado em cada atividade; $\mathrm{i}^{*}=$ Taxa Interna de Retorno

- A Relação Beneficio/Custo $(B / C)$ diz respeito a relação entre o valor presente dos benefícios e o valor presente dos custos, a uma dada taxa de juros ou descontos. Um projeto é considerado economicamente viável quando apresenta relação maior que 1 (VALE, 2004). Essa relação pode ser expressa através da fórmula abaixo:

A RBC pode ser calculada de acordo com a fórmula abaixo:

$$
R B C=\frac{B}{C}
$$




\section{RESULTADOS}

Na tabela abaixo apresentamos os custos que o proprietário obteve no preparo da área do plantio (Tabela 1).

Tabela 1. Custo de implantação de plantio de Teca em 1 hectare, no município de Santarém-Novo, Pará.

Table 1. Cost of implanting a Teak plantation in 1 hectare, in the municipality of Santarém-Novo, Pará.

\begin{tabular}{|c|c|c|c|c|c|}
\hline \multicolumn{6}{|c|}{ IMPLANTAÇÃO } \\
\hline \multirow{7}{*}{ 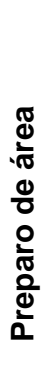 } & \multicolumn{4}{|c|}{ Ano 1} & \\
\hline & Variaveis & Unid. & & & \\
\hline & Gradagem & hora & 8 & 29,50 & 236,00 \\
\hline & Calagem (gesso) & ton & 1 & 160,00 & 160,00 \\
\hline & Calagem (calcário) & ton & 1,5 & 160,00 & 240,00 \\
\hline & Roçagem mecanizada & hora & 1 & 140,00 & 140,00 \\
\hline & & Total & & & $\mathrm{R} \$ 776,00$ \\
\hline \multirow{4}{*}{ 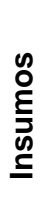 } & Formicidas & $\mathrm{kg}$ & 5 & 3,30 & 16,50 \\
\hline & Plantio e Replantio & & & & - \\
\hline & NPK & $\mathrm{kg}$ & 200 & 2,00 & 400,00 \\
\hline & & Total & & & $R \$ 416,50$ \\
\hline \multirow{4}{*}{ 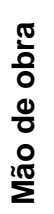 } & Calagem & diária & 3 & 140 & 420,00 \\
\hline & Capina Manual & diária & 6 & 32 & 192,00 \\
\hline & Cozinheira & diária & 6 & 35 & 210,00 \\
\hline & & Total & & & $\mathrm{R} \$ \mathbf{8 2 2 , 0 0}$ \\
\hline
\end{tabular}

Já na tabela 2, mostra-se os custos de manutenção do plantio que vai do segundo até o décimo quinto ano. 
Tabela 2. Custo de manutenção de plantio de Teca em 1 hectare, no município de Santarém-Novo, Pará.

Table 2. Maintenance cost of teak planting in 1 hectare, in the municipality of Santarém-Novo, Pará.

\begin{tabular}{ccccc}
\hline & \multicolumn{3}{c}{ MANUTENÇÃO } \\
\hline Váriaveis & Ano 2 & Valor Unit. & Valor Tot. \\
Herbicida & L & 20 & 19 & 380 \\
Roçagem mecanizada & hora & 1 & 140,00 & 140,00 \\
& Total & & & $\mathbf{5 2 0 , 0 0}$ \\
\hline Váriaveis & Unid. & Ano 3-15 & Valor Tot. \\
Roçagem mecanizada & hora & 13 & 140,00 & 1820,00 \\
& Total & & & $\mathbf{1 . 8 2 0 , 0 0}$ \\
\hline
\end{tabular}

$\mathrm{Na}$ Tabela 3, estão descritos os gastos com transporte e produtividade obtidos com o plantio.

Tabela 3. Valores referentes ao custo com transporte e produtividade de Teca no município de Santarém Novo, Pará.

Table 3. Values related to the cost of transportation and productivity of Teak in the municipality of Santarém Novo, Pará.

\begin{tabular}{cccc}
\hline UNIDADE & QUANTIDADE & \multicolumn{2}{c}{ Transporte } \\
$\mathrm{m}^{3}$ & 125 & VALOR UNITÁRIO (R\$) & VALOR TOTAL (R\$) \\
& & 80 & 10.000 \\
$\mathrm{~m}^{3}$ & 125 & Produtividade & 68.750 \\
\hline
\end{tabular}

O projeto possui viabilidade econômica quando as receitas são maiores que os custos. Entretanto, tal projeto obteve valor positivo somente no décimo sexto ano, semelhante ao estudo de Virgens et al. (2015), como mostra a tabela 5. 
Tabela 5. Fluxo de caixa para o plantio de Teca no município de Santarém Novo, Pará.

Table 5. Cash flow for planting Teak in the municipality of Santarém Novo, Pará.

\begin{tabular}{cccc}
\hline ANO & CUSTO (R\$) & RECEITA (R\$) & LUCRO (R\$) \\
\hline 1 & $2.486,00$ & 0 & $-2.486,00$ \\
2 & 520 & 0 & $-520,00$ \\
$3-15$ & $1.820,00$ & 0 & $-1.820,00$ \\
16 & $10.970,00$ & $68.750,00$ & $57.780,00$ \\
\hline TOTAL & $\mathbf{1 5 . 7 9 6 , 0 0}$ & $\mathbf{6 8 . 7 5 0 , 0 0}$ & $\mathbf{5 2 . 9 5 4 , 0 0}$ \\
\hline
\end{tabular}

A viabilidade do plantio de Teca pode ser visualizado pelos resultados dos indicadores econômicos na tabela 6.

Tabela 6. Valores de Indicadores Econômicos de plantio de Teca no município de Santarém Novo, Pará.

Table 6. Values of Economic Indicators of Teak planting in the municipality of Santarém Novo, Pará.

\begin{tabular}{cc}
\hline VPL & $\mathrm{R} \$ 9.969,28$ \\
VAE & $\mathrm{R} \$ 1.310,70$ \\
TIR & $20 \%$ \\
$\mathrm{~B} / \mathrm{C}$ & $\mathrm{R} \$ 10,59$
\end{tabular}

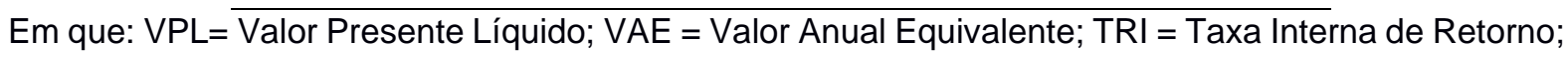
$\mathrm{B} / \mathrm{C}=$ Razão Benefício Custo.

Após análise do VPL, procedeu-se a uma análise de sensibilidade para 0 indicador Valor Anual Equivalente (VAE), o qual se obteve o valor de $R \$ 1.310,70$. Quanto a Taxa Interna de Retorno o empreendimento possui uma TIR de $20 \%$, estando maior que a taxa de desconto de $10 \%$, o que representa uma atividade viável financeiramente. Além destes, a Relação Custo/Benefício resultante no trabalho foi de $\mathrm{R} \$ 10,59$, apontando a viabilidade do projeto.

\section{DISCUSSÃO}

$\mathrm{Na}$ fase de manutenção do plantio, o proprietário teve somente gastos com 0 Herbicida Roundup e a roçagem mecanizada, totalizando um custo de $R \$ 2.340,00$ 
neste período. Quanto à manutenção, os custos se estabilizam do terceiro até o décimo quinto ano, pois só se fez necessário a roçagem mecanizada com intuito de reduzir a mato-competição, totalizando um custo de $\mathrm{R} \$ 140,00$ ano $^{-1}$.

Dessa forma, foi possível observar que os maiores custos ocorrem no $1^{\circ}$ ano de atividade em função do plantio e no 15 ano, quando são realizados desbastes, sendo corroborado por Sanguino (2009), que ao avaliar os custos de produção e a rentabilidade econômica por meio dos indicadores Valor Presente Líquido (VPL) e da Taxa Interna de Retorno (TIR) de um povoamento de Tectona grandis no município de Benevides no Pará, chegou à conclusão que os maiores custos foram neste períodos.

Além disso, também foi calculado o custo com transporte que foi de responsabilidade da empresa para qual o produtor vendeu as toras. $O$ valor que a empresa pagou para o transporte de toras de Santarém-Novo até o município de Dom Eliseu foi de $R \$ 80,00 \mathrm{~m}^{3}$, totalizando o custo de $R \$ 10.000$ nesta etapa.

Quando se trata das receitas do plantio, de acordo com os dados obtidos, esta possui uma produtividade de $125 \mathrm{~m}^{3} \mathrm{ha}^{-1}$ e o valor de $1 \mathrm{~m}^{3}$ foi de $\mathrm{R} \$ 550,00$, obtendose um total $R \$ 68.750$ com a comercialização do produto.

A viabilidade econômica do plantio de Teca pode ser visualizada pelos resultados dos indicadores econômicos, ou seja, VPL positivo e TIR maior que as taxas mínimas de atratividade (de 6\% a.a., 9\% a.a., e 12\% ao ano). O Valor Presente Líquido (VPL) para o plantio de teca em dezesseis anos foi de $\mathrm{R} \$ 9.969,28$, indicando que o investimento em questão é economicamente viável a juros de $10 \%$, em concordância com o trabalho de Tsukamoto et al. (2003). Entretanto, um estudo realizado por Bezerra et al. (2011) apresentou VPL negativo, a taxa de 10\%, no estado do Mato Grosso. De acordo com o autor, isso ocorreu devido ao baixo ritmo de crescimento da floresta frente aos altos custos de produção e ao valor agregado no momento da venda da madeira.

Através do indicador Valor Anual Equivalente (VAE) e da a Taxa Interna de Retorno, é possível afirmar que o projeto é economicamente viável com resultado próximo ao VAE constatado em trabalho realizado por Marques et al. (2016) de um plantio florestal de teca e mogno, no sudeste do Pará. Em analise comparativa, 
apresentou TIR inferior a encontrada por Macena et al. (2015) sobre sistema silvipastoril com Paricá para agricultura familiar do nordeste paraense onde o valor obtido foi de 31,04\%. Contudo, apresentou TIR maior que aquela encontrada em trabalho realizado por Martin (2017), com Pinus elliottii, no sul do país correspondente a $13,54 \%$.

A Relação custo/benefício também apontou para a viabilidade do projeto, visto que o critério de decisão para considerar o investimento viável é a relação benefício/custo maior que 1 (SÁ et al., 2010). Neste mesmo trabalho sobre o cultivo de teca para produção de madeira, a $R C B$ foi igual a $R \$ 1,17$, o que mostra superioridade significativa do plantio em Santarém Novo.

\section{CONCLUSÕES}

A partir da análise de dados desse projeto, pode-se concluir que, 0 empreendimento é viável economicamente. No entanto, apesar de possuir altos valores de lucratividade, com baixos custos de implantação, o plantio só apresentou retorno financeiro no último ano. Portanto, pode-se inferir que os plantios florestais com teca, são ótimas opções de investimento, funcionando como alternativas rentáveis e sustentáveis a empreendedores do ramo de base florestal, bem como oportunidade na conquista de mercados exigentes por produtos florestais de qualidade.

\section{REFERÊNCIAS}

BEZERRA A. F.; MILAGRES, F. R.; SILVA, M. L.; LEITE H. G. Análise da viabilidade econômica de povoamentos de Tectona grandis submetidos a desbastes no Mato Grosso. Cerne, v. 17, n. 4, p. 583-592, 2011.

BHATIA, V.; SRIVASTAVA, S. P.; SRIVASTAVA, R.; MISHRA, A.; NARENDER, T.; MAURYA, R.; SRIVASTAVA, A. K. Antihyperglycaemic and aldose reductase inhibitory potential of Acacia catechu hard wood and Tectona grandis leaves. Medicinal Chemistry Research, v. 20, p. 1724-1731, 2011. 
COSTA, R. B. C.; RESENDE, M. D. V.; SILVA, V. S. M. Experimentação e seleção no melhoramento genético de Teca (Tectona grandis Lf). Floresta e Ambiente, v. 14, n. 1, p. 76-92, 2012.

FLÓREZ, J. B. Simulação em serviço de pisos de madeira jovem de Tectona grandis. Revista Matéria, v. 20, n. 4, p.1048-1060, 2015.

KLIPPEL, V. H. Zoneamento climático para teca, cedro australiano, nim indiano e pupunha no estado do Espírito Santo. Floresta, v. 43, n. 4, p. 671-680, 2013.

MACENA, R. P.; DE AZEVEDO, C. M. B. C.; KATO, O. R. Viabilidade econômica de um sistema silvipastoril para agricultura familiar do nordeste paraense. In: Seminário de Iniciação Científica, Seminário de Pós-Graduação da Embrapa Amazônia Oriental. Belém, Pará, 2015.

MARQUES, M. C.; QUEIROZ, J. F. Modelagem econômica de sistema agroflorestal para agricultores familiares no sudeste do Pará. Cadernos de Agroecologia, v. 10, n. 3, 2016.

MARTIN, S. S. Análise física e econômica de dois sistemas de manejo de Pinus elliottii Engelm em duas áreas do sul do Brasil. Universidade Federal do Paraná, 2017, 9p.

MORA, F.; HERNÁNDEZ, W. Estimación del volumen comercial por producto para rodales de teca en el pacífico de Costa Rica. Agronomía Costarricense, v. 31, n. 1, p. 101-112, 2007.

NOCETTI, M.; ROZENBERG, P.; CHAIX, G.; MACCHIONI, N. Provenance effect on the ring structure of teak (Tectona grandis L. f.) wood by X-ray microdensitometry. Annals of forest science, v. 68, n. 8, p. 1375-1383, 2011.

PAES, J. B.; SANTOS, L. L.; SILVA, L. F.; MOTTA, J. P.; BRAZ, R. L.; LOMBARDI, R. R. Caracterização tecnológica da madeira juvenil de teca (Tectona grandis) visando à produção de móveis. Revista Brasileira de Ciências Agrárias, v. 10, n. 3, p. 437-442, 2015.

PElissaRI, A. L.; GUIMARÃES, P. P.; BEHLING, A.; EBLING, A. A. Cultivo da teca: características da espécie para implantação e condução de povoamentos florestais. Agrarian Academy, v. 1, n. 1, p. 127-145, 2014.

PREFEITURA MUNICIPAL DE SANTARÉM NOVO. Cidade Santarém-Novo. Disponível em: <http://www.santaremnovo.pa.gov.br/pg_historia/pg_historia.php>. Acesso em 08/01/2019. 
RAMACHANDRAN, S.; RAJASEKARAN, A.; KUMAR, K. M. Antidiabetic, antihyperlipidemic and antioxidant potential of methanol extract of Tectona grandis flowers in streptozotocin induced diabetic rats. Asian Pacific Journal of Tropical Medicine, p. 624-631, 2011.

REZENDE, J. L. P. Análise econômica de fomento florestal com eucalipto no estado de Minas Gerais. Cerne, v. 12, n. 3, p. 221-231, 2006.

SÁ, C. P.; FIGUEIREDO, E. O.; OliVEIRA, L. C. Caracterização e análise da rentabilidade financeira do cultivo da teca (Tectona grandis Lf) para produção de madeira em Rio Branco, Acre. Embrapa, Rio Branco, 2010, 6p.

SANGUINO, A. C. Custos de implantação e rentabilidade econômica de povoamentos florestais com teca no estado do Pará. Revista de Ciências Agrárias, v. 52, n. 1, p. 61 78, 2009.

TSUKAMOTO, A. F. A.; SILVA, M. L.; COUTO, L.; MULLER, M. D. Análise econômica de um plantio de teca submetido a desbastes. Revista Árvore, v. 27, n. 4, p. 487-494, 2003.

VALE, R. S. Agrossilvicultura com eucalipto como alternativa para o desenvolvimento sustentável da zona da mata de Minas Gerais. 2004. 115 f. Tese (Doutorado em Ciências Florestais) - Universidade Federal de Viçosa, Viçosa, 2004.

VIRGENS, A. P.; FREITAS, L. C.; LUZ, D. S.; MOREIRA, A. C. D. Análise econômica e de sensibilidade em projetos de reflorestamentos no Estado da Bahia. Enciclopédia Biosfera, Centro Científico Conhecer, v. 11, n. 21, p. 120-27, 2015.

VIRGENS, A. P.; FREITAS, L. C.; LEITE, A. M. P. Análise Econômica e de Sensibilidade em um Povoamento Implantado no Sudoeste da Bahia. Floresta e Ambiente, v. 23, n. 2, p. 211-219, 2016. 